\title{
La faune de l'Afrique du Nord et du Sahara d'après Hérodote
}

\author{
Gabriel Camps *
}

Combien d'erreurs manifestes, de contradictions, d'invraisemblances ont été, au cours des siècles, dénoncées dans l'oeuvre d'Hérodote. Récemment une thèse brillante de F. Hartog (1980) a mis en lumière certains aspects de l'imaginaire chez l'historien d'Halicarnasse sur sa conception de l'Autre, du Barbare, sur la notion du Nomade opposé au Laboureur dont le Grec du $V^{e}$ siècle est infailliblement le modèle. Les nouvelles lectures proposées, si elles rendent justice à Hérodote, risquent cependant de réduire d'autant l'apport propement documentaire d'une oeuvre immense (G. Camps, 1985).

Je ne présenterai aujourd'hui que quelques commentaires sur ce que Hérodote nous apprend de la faune de la Libye. Si nous suivons scrupuleusement l'ordre choisi par Hérodote (IV, 191-192) nous sommes d'abord en présence d'une liste des animaux sauvages qui vivent dans le pays occupé par les Libyens laboureurs, c'est-à-dire ce que nous appelons le Maghreb, plus spécialement sa partie tellienne, celle qui se situe à l'occident du Tritonis, pays montagneux et couvert de forêts. Nous y trouvons:

- Des serpents de très grande taille. A cette citation semblent faire écho des récits légendaires plus récents, reflets de l'importance du serpent dans l'imaginaire des populations nord-africaines à toutes les époques; on songe en particulier au "dragon" contre lequel l'armée de Regulus dut livrer un véritable combat sur les bords du Bagradas (TiteLive, Perioch. XVIII, Pline l'Ancien, VIII, 37), précisément dans une région où fut pratiqué, à l'époque romaine, un culte de Draco (Thignica, CIL,

\footnotetext{
* Université de Provence (LAMPO).
} 
VIII, 15247; Numluli, ibid. 15378). Pline rapporte que la peau du dragon du Bagradas, exposée à Rome, aurait mesuré 120 pieds (soit $35 \mathrm{~m}$ ). En réalité, les plus grands serpents du Maghreb sont les couleuvres de Montpellier. Le plus grand sujet de cette espèce, qui fut tué à Goulimine (Sud marocain), atteignait $3 \mathrm{~m}$. Des serpents de $3 \mathrm{~m}$. de long sont, certes, de grands serpents, mais ils ne méritent guère le qualificatif d'énormes (hypermégas) que leur impose Hérodote.

- Des lions. Je n'aurai aucun commentaire à faire sur ce fauve qui fut abondant dans toute l'Afrique du Nord pendant toute le durée des temps historiques, de l'Antiquité jusqu'au XIX ${ }^{\mathrm{e}}$ siècle.

- Des éléphants. Ils ne disparurent que sous la domination romaine. Elephas (Loxondota) atlanticus est une sous espèce de l'éléphant d'Afrique qui est plus grand et vit dans un biotope différent.

- Des ours. La présence de Ursus arctos en Afrique dans l'Antiquité, fait probléme. Deux espèces d'ours ont vécu durant les temps paléolithiques en Afrique du Nord (seule partie du continent à connaître des ursidés et des cervidés); elles semblent y avoir pénétré pendant la glaciation de Riss, mais l'ours ne figure jamais dans l'art rupestre néolithique; on ne trouve guère, non plus, d'ossements qui lui soient atribuables dans les gisements néolithiques. On peut croire qu'il a disparu à cette époque et le fait semble confirmé par Pline qui précise que cet animal ne vit pas en Afrique (VIII, 228), ce qui ne l'empêche pas de mentionner des “ours de Numidie» (VIII, 131) parmi les fauves qui combattent au cirque, à Rome, en 61 av. J.-C. Au $V^{e}$ siècle de notre ère, cet animal vivait encore en Afrique, apparemment, puisque saint Augustin en vit dans les jeux offerts à Thagaste (Sermo. XXXII, 20). Malgré l'assertion de Pline, de nombreux auteurs confirment la présence de l'ours en Afrique; $S$. Gsell ajoute qu'il figure souvent dans les mosaïques africaines (S. Gsell, 1913: 115) mais celles-ci représentent, mêlées à des espèces authentiquement africaines, des animaux asiatiques comme le tigre, le paon... II n'est pas impossible que dans certains cas il y ait eu confusion entre l'ours (qui compte tenu des conditions naturelles devait être de plus petite taille que l'ours brun européen) et l'hyène rayée; on pourrait même se demander si l'ours dit de Numidie n'était pas une hyène; cet animal n'a disparu du Maghreb qu'à l'époque contemporaine, il existe encore dans le Sahara, et peut-être dans certains secteurs de l'Atlas marocain. Toutefois Hérodote cite, à la fois, les ours chez les Libyens sédentaires et l'hyène chez les Nomades; cette précision est donc de grande importance.

- Aspics. II s'agit soit de la vipère, très commune au Maghreb, soit du cobra naja, espèce plus rare mais encore connue aujourd'hui et présentée par les charmeurs de serpents. 

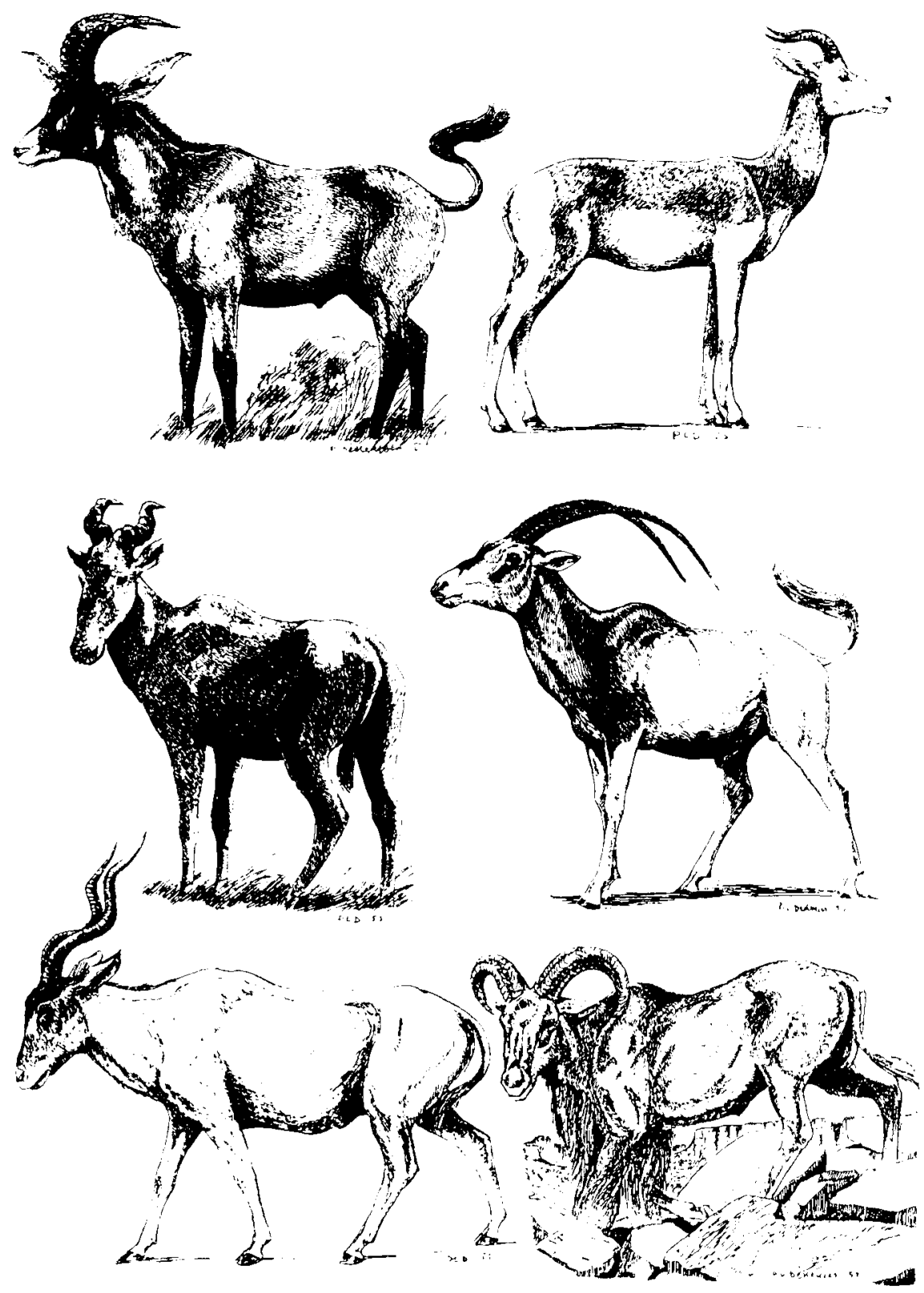

Fig. 1. Quelques ruminants d'Afrique cités par Hérodote: I'Hippotrague, la Gazelle mohor, l'antilope Bubale, l'Oryx, l'Addax, le Mouflon à manchettes (dessins de P. L. Dekeyser). 
- Anes pourvus de cornes. II s'agit bien évidemment de l'antilope chevaline, Hippotragus equinus, dont le port et le galop ainsi que les formes rappellent invinciblement ceux d'un équidé. L'hippotrague est connu à l'état fossile dans plusieurs gisements paléolithiques (Sidi AbderRahmane, Dar es-Soltan, Kifan bel Ghomari...); il vit encore au Sahara méridional. D'autres hippotraginés, comme l'antilope bubale mentionnée par Hérodote chez les Libyens nomades, peuvent être comptées parmi ces "ânes à cornes" (fig. 1).

- Monstres à tête de chien (Cynocéphales) ou dépourvus de tête (Acéphales) et un grand nombre d'autres bêtes, lesquelles, ajoute malicieusement Hérodote, ne sont pas fabuleuses (sous-entendu comme les monstres qui viennent d'être cités) ce qui montre que l'historien-rapporteur n'est pas dupe et qu'il sait faire la part de la hâblerie, même s'il reproduit fidèlement ses sources.

Un premier commentaire s'impose à la lecture de cette liste faunistique de la Libye occidentale. Remarqueons son désordre, Hérodote ne tente même pas de citer, côte à côte, les animaux qui se ressemblent ou qui vivent dans le même biotope. Lions, éléphants et ours s'intercalent entre deux variétés de serpents, ceux de très grande taille et les aspics.

Autre remarque: cette liste est très incomplète. On note des absences surprenantes, tant ces animaux sont caractéristiques du paysage faunique africain: le mouflon, le chacal, l'âne sauvage qui figurent dans l'art rupestre néolithique et dont les ossements se retrouvent dans les gisements préhistoriques. Durant l'Antiquité ils demeurent abondants et sont souvent cités. Fait aussi défaut le magot (Macaca inuus), le cercopithèque du Maghreb, qui n'est pas cité nommément par Hérodote; mais celuici écrit quelques lignes plus bas (IV, 194) que les Gyzantes, voisins des Zauèkes que nous situerons volontiers en Zeugitane, mangent des singes qui sont très nombreux dans leurs montagnes.

Hérodote ne cite, non plus, ni le cerf ni le sanglier; il précise même plus loin, dans l'étude de la faune de la Libye des nomades, que ces animaux font complètement défaut dans toute la Libye. Or si le cerf de Barbarie (Cervus elaphus barbarus) a toujours été assez rare et cantonné dans les secteurs les plus humides du Tell, aux confins de l'Algérie orientale et de la Tunisie, le sanglier est abondant partout, jusque dans l'Atlas saharien. II s'agit donc là d'une erreur manifeste d'Hérodote.

Cette première liste concernant la faune maghrébine pèche donc par défaut, mais Hérodote en est parfaitement conscient aussi prend-il soin de terminer sa recension par un complément collectif: "et un grand nombre de bêtes qui, elles, ne sont pas fabuleuses». A vrai dire, la minceur 
de cette liste, les rajouts merveilleux, les erreurs sur l'absence du cerf et du sanglier ne sont que le reflet de l'ignorance générale d'Hérodote sur les Libyens cultivateurs, ceux qui, au-delà du Tritonis, habitent dans un pays montagneux et dont il ne peut citer que trois tribus, les Maxyes, les Gyzantes et les Zauèkes. Ces peuples paraissent d'autant plus «barbares" qu'ils sont méconnus; le monde punico-berbère se situe au-delà du front pionnier de la connaissance hellénique qui ne dépasse guère les rives de la Petite Syrte.

Passant à la description de la faune du pays des Libyens nomades, c'est-à-dire de la Libye à l'orient du Tritonis, en gros le territorie de l'actuel Etat libyen, Hérodote se montre plus disert et plus précis. Voici, sans chercher à les identifier, les animaux qu'il cite: pygargues, zorcades, bubales, ânes sans cornes, oryes, bessaria, hyènes, porcs-épics, béliers sauvages, dictyes, chacals, panthères, boryes, crocodiles terrestres, autruches, petits serpents ayant une corne sur le tête, dipodes, zégéries, hérissons, galai. Cette liste, plus abondante que la précédente, alors que la faune saharienne est plus pauvre que celle du Maghreb est présentée selon un certain ordre. Nous verrons que les cinq premiers animaux sont des herbivores, viennent ensuite des animaux «agressifs", carnivores comme le renard (bessaria), le chacal, la panthère, parmi lesquels le porc-épic trouve, peut-être sa place en raison de ses piquants et le bélier sauvage c'est-à-dire le mouflon, peut-être en raison de la grosseur et de la taille de ses cornes; Hérodote cite ensuite des animaux spécifiques de la région: crocodiles terrestres, autruches, petits serpents à une corne, puis trois sortes de «rats" et enfin l'animal qu'il appelle gala qui est identique à celui qui vit dans la région de Tartessos.

Presque toutes les espèces citées sont identifiables.

- Pygargue. Ce n'est pas le rapace qui porte aujourd'hui ce nom mais la gazelle dama, ou gazelle mohor, connue aussi sous le nom de Biche Robert, très reconnaissable à sa "culotte blanche". En Mauritanie cette belle antilope est curieusement appellée «Emir Trarza» parce qu'elle a un pantalon blanc, comme celui de l'Emir du Trarza qui seul avait droit de porter un tel vêtement.

- Zorcades. II faut lire dorcades. Ce sont les gazelles de petite taille, les plus communes au désert.

- Bubales, ou plus exactement antilopes bubales qui subsistèrent au Maghreb jusqu'au milieu du XIX siècle. L'antilope bubale (Alcelaphus boselaphus) était désignée sous l'appelation de "Beguer el Ouach" (la vache sauvage), bien que son allure générale, sa très longue face, son garrot surélevé la différencient de bovinés. 
- Anes. Que sont ces ânes, ceux qui n'ont pas de cornes et qui ne boivent pas? II s'agit des ânes sauvages, des onagres, qui ont vécu au Maghreb et au Sahara pendant les temps préhistoriques et l'Antiquité et sur lesquels les écrivains nous ont laissé de nombreux témoignages. Les prétendus ânes sauvages actuels du Hoggar et du Sahara central ne sont que des ânes marrons. Mais, avec la liberté, ces animaux ont recouvré une fière allure et des flancs rebondis qui étonnent dans un tel monde minéral. On les distingue facilement des misérables bourricots des campements du désert et des villages du Tell. Ces ânes marrons ne sont pas limités dans leurs déplacements par le souci de rester proches d'un point d'eau; lorsque les coloquintes sauvages arrivent à maturité dans les lits sableux d'oueds fantômes, ils trouvent dans ces fruits les quantités d'eau suffisantes, pendant un certain temps, à leur équilibre biologique, d'où l'assertion quelque peu exagérée d'Hérodote mais reposant sur un fait exact.

- Oryes. Gsell propose de lire oryx, mais selon lui l'animal, dont les cornes servaient d'après Hérodote à faire des lyres phéniciennes, ne serait pas l'oryx mais l'addax. Cette antilope a en effet la taille d'un petit boeuf, comme l'indique Hérodote, et surtout ses cornes sont lyrées alors que celles de l'oryx (Oryx leucoryx) sont très longues (elles dépassent un mètre) et ont une courbure simple. Mais contrairement à ce que croyait $\mathrm{S}$. Gsell, l'oryx, antilope des pays arides, pouvait très bien vivre dans le pays des Nasamons et des Garamantes; il n'a disparu que très récemment du Sahara septentrional. Si ces cornes ne sont pas lyrées elles peuvent néanmoins entrer facilement dans la fabrication de lyres de grandes dimensions dont les montants sont droits ou à simple courbure. Notons que l'oryx vit aussi dans le désert arabique où les Phéniciens pouvaient s'approvisionner en cornes de cette antilope pour fabriquer leurs lyres. Nous maintiendrons l'oryx dans la liste donnée par Hérodote, tout en reconnaissant que l'addax avait autant de chance d'y figurer.

Bassaria. Ce sont de petits renards dont le nom s'est conservé chez les Coptes. On songe en premier lieu au gracieux fennec (Fennec zerda), mais il existe aussi un renard proprement saharien (Vulpes Rupelli) de taille inférieure à celle du renard commun et dont les oreilles sont moins démesurées que celles du fennec.

Hyénes et porcs-épics ne méritent pas de commentaires particuliers, ce sont des animaux vivants actuellement au Sahara.

Béliers sauvages. Par cette expression Hérodote désigne évidemment les mouflons à manchettes, commettant ainsi une erreur très répandue puisque le mouflon d'Afrique n'est pas un ovin mais appartient à un 
genre dont il constitue l'espèce unique (Ammotragus lervia) plus proche des caprins que des ovins.

Dictyes, comme Boryes (à moins qu'il ne s'agisse d'un doublet fautif d'Oryes) résistent á toute identification.

Chacals. Ils sont communs dans tout le nord de l'Afrique.

Panthères. On les attendait plutôt dans la Libye montagneuse et forestière où elles subsistèrent jusqu'a à la fin du siècle dernier; mais comme le suggère $S$. Gsell, Hérodote pense peut-être au guépard que les auteurs anciens ne distinguent guère de la panthère. Le guépard, qui chasse dans les endroits dégagés, vit encore au Sahara. II est le prédateur habituel des gazelles.

Crocodile terrestre. Cet animal est mal nommé mais fort bien décrit; il mesure 3 coudées et ressemble beaucoup au lézard: il s'agit du varan (Varanus niloticus) dont la longueur atteint $1,50 \mathrm{~m}$, ce qui est supérieur aux dimensions données par Hérodote.

Autruches. Elles ont vécu dans le nord du Sahara jusqu'au début de notre siècle.

Serpents de petite taille ayant une corne sur la tête. Ils semblent devoir être identifiés aux cérastes, vipères à cornes communes au Sahara mais le fait qu'il s'agisse de corne unique indique plutôt une autre espèce saharienne, la vipère heurtante (Bitis arietans) qui porte une écaille saillante sur le museau.

Dipodes. Hérodote mentionne ensuite trois sortes de "rats", dont le hérisson qui est un insectivore et non un rongeur. Les dipodes sont des gerboises, la grande gerboise (Jaculus jaculus) plutôt que la gerbille (Gerbillus gerbillus) plus discrète et de plus petite taille.

Zégéries. C'est une autre espèce de «rat». Hérodote dit que le nom de Zégéries est libyque et répond au nom grec qui signifie colline. Gsell fait un procès à notre auteur sur ce propos. Voici son argumentation: dans une scolie de Dioscoride (IV,123), il est précisé que "zigar» est un nom punique désignant une plante nommée en grec "bounion" (c'est-àdire "de colline»). Gsell en déduit qu'Hérodote ne connaissait pas la véritable origine phénicienne de ce mot. "ll est donc probable, conclut-il, qu'il l'a recueilli... dans une conversation avec un Cyrénéen ou bien dans quelque ouvrage grec". Je ne vois pas pourquoi il faudrait accorder une plus grande créance à un commentaire de Dioscoride qu'à une assertion d'Hérodote. On sait combien est ambiguë la notion de punique (C. Courtois, 1950; G. Camps, 1979). Sous cette expression peut fort bien se 
cacher une origine proprement libyque, c'est-à-dire berbère. On retrouve, en effet, dans le nom des zégéries un radical pan-berbère ZGR qui signifie «rouge». Ce radical apparaît dans le nom touareg des cultivateurs à peau sombre des oasis: Izaganen. Ces cultivateurs se disent "rouges" pour ne pas être confondus avec les esclaves noirs. De même une tribu maure de l'Antiquité, dans le nord du Maroc actuel, portait un nom semblable transcrit sous la forme Zegrenses en latin (M. Euzennat, 1974).

Comment expliquer l'apparition d'un autre sens, celui de colline, retenu à la fois par Hérodote et par Dioscoride? II existe en berbère une autre racine très proche de la précédente $Z G R$, qui sous la forme féminine «tazeggarwt» désigne le jujubier sauvage (M. Dallet, 1982: 936) qui comme le "bounion" est aussi une plante de colline.

En définitive, nous avons deux identifications possibles, les zégéries seraient des "rats rouges" ou des "rats de colline" (c'est-à-dire de rochers). Dans le premier cas on songerait à l'écureuil terrestre ou fouisseur (Xerus gaetulicus), dans le second au goundi (Ctenodactylus gundi). Ma préférence va au goundi car cet animal est plus répandu que l'écureuil terrestre et vit en colonies plus nombreuses; il a de plus un pelage brun-rouge. II est donc à la fois de rocher et de couleur rougeâtre. Enfin il a une chair très estimée (A. Louis, 1975: 194) et a donc plus de chance d'avoir retenu l'attention des informateurs d'Hérodote (fig. 2).

Galai. Ces animaux, qui prospèrent dans le pays ou pousse le sylphium, ressemblent, dit Hérodote, à ceux qui vivent à Tartessos, donc dans le sud de la Péninsule Ibérique. II semble que sous ce nom les Anciens aient confondu plusieurs espèces. D'après Strabon, il s'agit d'un animal ayant l'aspect d'un chat mais avec un museau pointu; cette description convient parfaitement á la genette qui vit á la fois en Europe et en Afrique. Un autre passage de Strabon (S. Gsell, 1913: 114), indique que des animaux sauvages africains portant le même nom étaient utilisés par les Espagnols pour poursuivre les lapins dans leurs terriers; il s'agirait ici de furets. Nous retiendrons la première identification, car la genette (Genetta genetta) est le viverridé le plus répandu en Afrique du Nord.

Parmi les animaux domestiques cités par Hérodote, se trouve une variété particulière de boeufs dont la description plus que toute autre révèle apparemment la naïveté de l'auteur. Parlant des Garamantes, dont nous connaissons la localisation au Fezzan et aux confins du Tassili n'Ajjer, il écrit: (traduction S. Gsell) "on trouve chez eux des boeufs qui

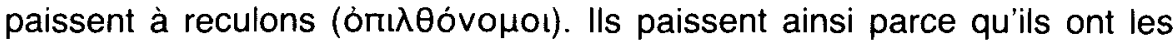
cornes inclinées en avant, ce qui les force á aller á reculons quand ils 


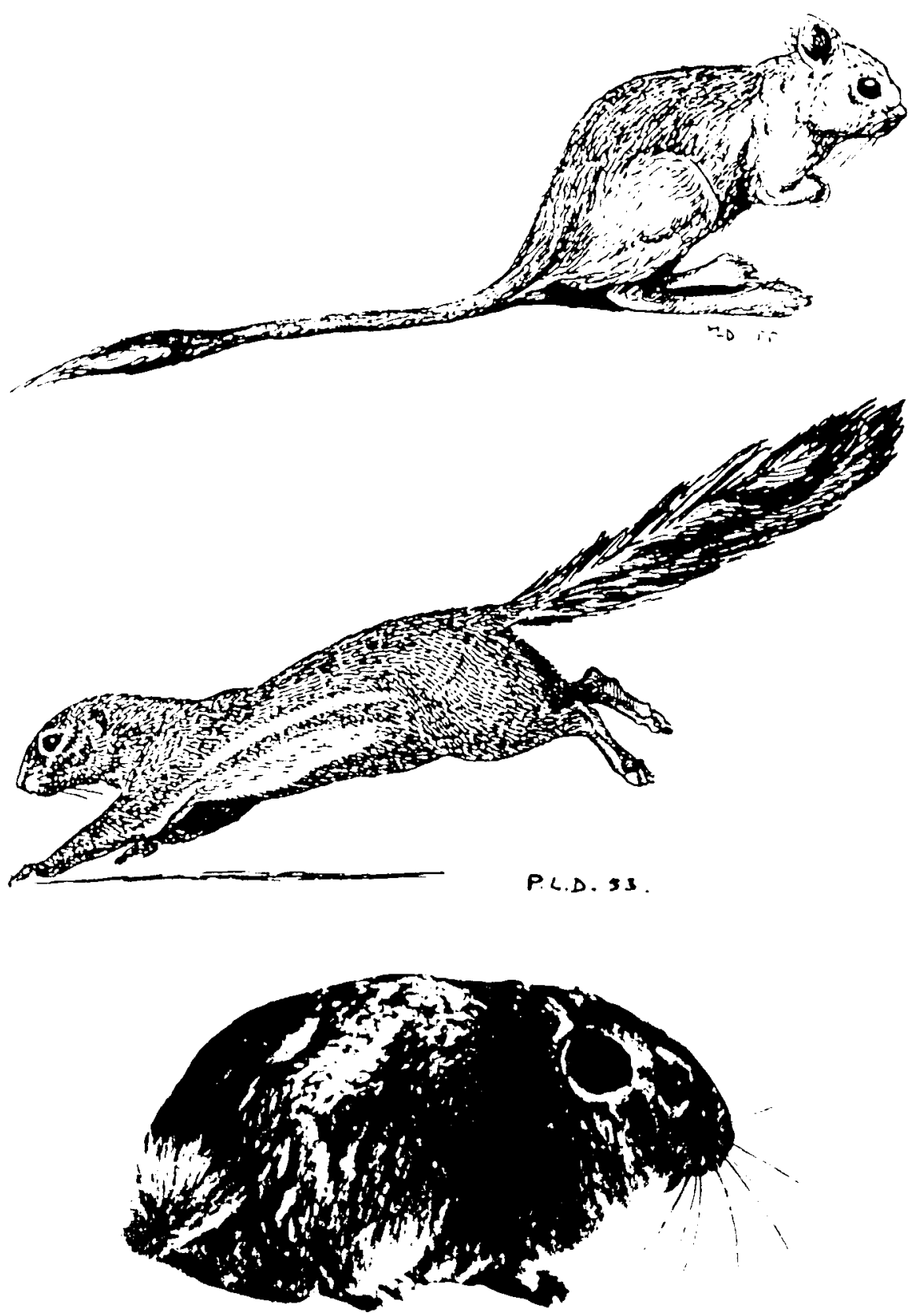

Fig. 2. Trois rongeurs sahariens: la Gerboise, l'Ecureuil terrestre, le Goundi (dessins de P. L. Dekeyser). 
paissent; ils ne peuvent marcher en avant car leurs cornes s'enfonceraient dans la terre...” (IV,183).

Comment prendre au sérieux une telle affirmation? Quelle que soit l'inclinaison des cornes vers l'avant et leur longueur, jamais la disposition serait telle que l'animal serait contraint de brouter à reculons, d'autant plus que, privé d'incisives au maxillaire supérieur, il lui aurait été impossible de trancher l'herbe dans un mouvement de la tête d'avant en arrière. Si Pomel avait cru pouvoir créer une espèce qu'il baptisa "Bos opisthonomus" qui n'est qu'une variété de Bos primigenius, il n'était pas dupe de l'assertion d'Hérodote; ce n'était qu'en raison de la courbure particulière de la corne (nullement gênante pour paître) qu'il avait créé cette espèce qui n'est plus retenue aujourd'hui.

Pourquoi Hérodote a-t-il rapporté ce que nous sommes bien tentés d'appeler une légende? Le récit n'est pas sans rappeler le rapt des génisses d'Apollon par Hermès qui les contraignait à marcher à reculons pour tromper les éventuels poursuivants mais, mis à part la locomotion rétrograde, aucune analogie n'apparaît entre les deux contes. S'agit-il bien d'un conte? Une meilleure connaissance de l'art rupestre saharien nous permet aujourd'hui de suggérer une explication. On sait que durant le Néolithique et encore au début de l'Age des métaux, jusqu'en pleine période du cheval, les Sahariens, particulièrement ceux qui fréquentaient les vallées du Tassili n'Ajjer, ont figuré d'importants troupeaux de boeufs domestiques. Ces gravures et ces peintures sont d'un réalisme et d'une qualité esthétique tels qu'il est possible de reconnaître sans peine la variété de boeuf représenté, il s'agit du "Bos africanus", un animal de grande taille, élancé, à la robe souvent pie ou tricolore, remarquable surtout par ses cornes, longues, effilées et lyrées chez les femelles, plus épaisses et plus courtes chez les taureaux où elles pointent vers l'avant. Or, parmi ces représentations si fidèles, on remarque certaines anomalies: des bêtes qui, comme dans les fresques égyptiennes, sont dépourvues de cornes et d'autres qui ont des cornes "flottantes" ou "ballantes". II semble que la cheville osseuse ait été attaquée à la base par un agent extérieur à moins que cette anomalie soit le résultat d'une carence alimentaire ou plus simplement d'une tare génétique. II n'est pas impossible que le "flottement" de la corne ait précédé la chute et la disparition de celle-ci. Dans une fresque de Jabbaren (Tassili n'Ajjer), on voit côte à côte des bovins à cornes lyrées normales, deux à cornes flottantes et deux dépourvus de cornes (fig. 3).

Quand on examine ces figures on comprend que les bovins à cornes flottantes devaient être gênés pour brouter puisque les cornes tombent de part et d'autre et que la pointe se trouve à quelques centimètres en 

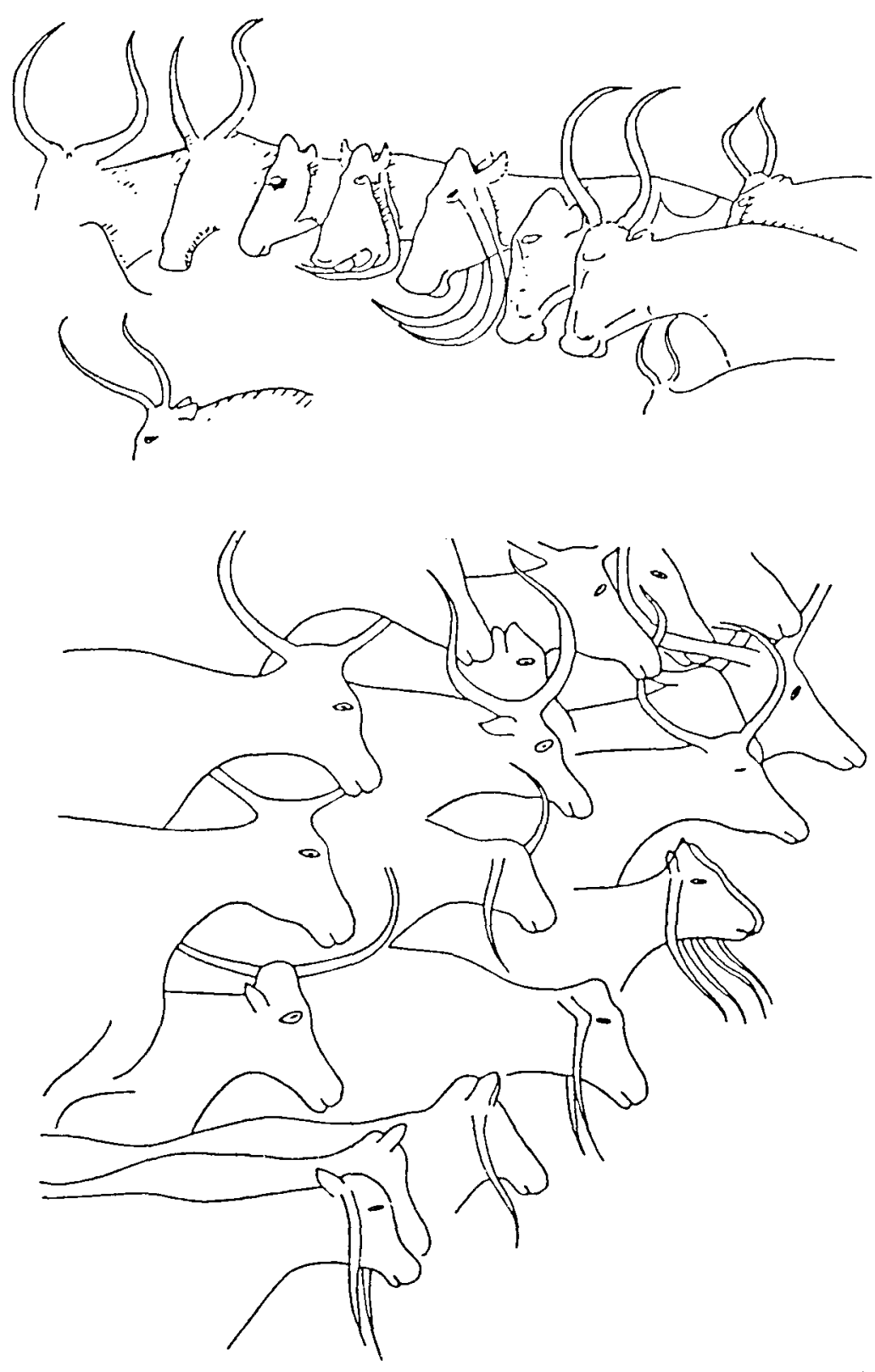

Fig. 3. Bovins des peintures rupestres du Tassili n'Ajjer, en haut station de Jabbaren (d'après Brenans), en bas de l'oued Derbaouen (d'après Colombel). Plusieurs animaux ont les cornes ballantes. 
avant du mufle. Penser qu'une telle disposition oblige l'animal à paître à reculons, la déduction n'est pas si sotte. J'imagine volontiers quelque scène de cabaret au cours de laquelle un caravanier garamante, décrivant aux citadins grecs ou libyens du littoral les belles fresques peintes par ses aïeux ou ses prédécesseurs dans les vallées du Tassili, insiste, au cours du récit, sur un détail qui a retenu son attention: la représentation de ces boeufs dont «les cornes inclinées en avant les forcent à aller à reculons quand ils paissent" (IV, 183). Le bruit en est arrivé jusqu'à Hérodote qui le consigna sur ses tablettes.

Cette recension des animaux libyques, nommés ou décrits par Hérodote, et pour laquelle nous sommes étroitement tributaires des excellents commentaires de S. Gsell, peut paraître un sujet particulièrement mince et de faible intérêt, dans la mesure où l'archéologie pré et protohistorique nous apporte une documentation plus précise sur la faune de ces contrées. Cependant dans cette faune ou ce bestiaire d'Hérodote, nous avons pu tout au long du texte retenir des notations justes bien que surprenantes (les ânes sauvages qui ne boivent pas), des tentatives d'explication rationnelle de phénomènes inhabituels (les boeufs opisthonomes des Garamantes), de vrais soucis philologiques ou etymologiques (à propos des zégéries), le choix du mot juste qui fait image (les "culs-blancs" ou Biche Robert, les dipodes ou gerboises) le désir d'informer (les oryx dont les cornes servent à fabriquer des lyres phéniciennes, ou les genettes du pays du silphium qui sont semblables à celles de Tartessos). II lui arrive, certes, de se laisser entraîner à quelques exagérations (les serpents énormes et de très grande taille, mais nous avons vu que la taille du varan est plutôt sous-estimée); il lui arrive aussi de verser dans la fable, et s'il cite quelques monstres dans son bestiaire (deux lignes du chapitre 191), du moins pred-il soin de dire qu'il ne s'agit que de propos de Libyens et il ajoute que les autres animaux, qu'il ne cite pas, ne sont pas "nés du mensonge". Mais sans être dupe de ses informateurs, Hérodote ne peut résister au plaisir de rapporter légendes ou historiettes et cet état d'esprit apparaît même dans une énumération aussi pauvre et sévère que celles des animaux de la Libye. Cette tentation, à laquelle il succombe avec délice, là beaucoup desservi auprès des esprits chagrins; nous la lui pardonnons volontiers. Somme toute, Hérodote avait une âme de journaliste. 


\section{BIBLIOGRAPHIE}

CAmps, F., 1979: Les Numides et la civilisation punique. Antiquités africaines, 14: 43-53.

CAMPS, G., 1985: Pour une lecture naïve d'Hérodote. Les récits libyens (IV, 168-199). Histoire de l'Historiographie, 7: 38-59.

Curtois, C., 1950: Saint Augustin et la survivance du punique. Revue africaine, 94: 259-282.

-DALLET, J. M., 1982: Dictionnaire kabyle-français. Cellaf, Paris.

EUZENNAT, M., 1974: Les Zegrenses. Mélanges d'histoire ancienne offerts à W. Seston: 175-186.

GSELL, S., 1913: Histoire ancienne de l'Afrique du Nord, t. 1. Hachette, Paris.

GSELL, S., 1916: Hérodote. Textes relatifs à l'Histoire ancienne de l'Afrique du Nord. Alger, Jourdan.

HaRtog, F., 1980: Le miroir d'Hérodote. Essai sur la représentation de l'autre. Gallimard, Paris.

LouIs, A., 1975: Tunisie du Sud. Ksars et villages de crêtes. CNRS, Paris.

MONTEIL, V., 1951: Contribution à l'étude de la faune du Sahara occidental. Institut des Hautes études marocaines, IX. 
